\title{
Decision-making at initial surgery for type A aortic dissection in patients with Marfan syndrome: proximal or extensive repair
}

\author{
Ning Li", Yu Zhang", Yuan Gao", Yifan Bai, Zhao An, Guanxin Zhang, Qingqi Han, Fanglin Lu, \\ BaiLing Li, Lin Han, Zhiyun Xu \\ Department of Cardiothoracic Surgery, Changhai Hospital, Second Military Medical University, Shanghai 200433, China \\ Contributions: (I) Conception and design: Z Xu, N Li; (II) Administrative support: Z Xu, L Han, F Lu; (III) Provision of study materials or patients: \\ Z Xu, B Li; (IV) Collection and assembly of data: N Li ,Y Zhang, Y Gao, Y Bai, Z An; (V) Data analysis and interpretation: G Zhang, Q Han; \\ (VI) Manuscript writing: All authors; (VII) Final approval of manuscript: All authors. \\ \#These authors contributed equally to this work. \\ Correspondence to: Zhiyun Xu. Department of Cardiothoracic Surgery, Changhai Hospital, Second Military Medical University, Shanghai 200433, \\ China. Email: 1378174246@qq.com.
}

Background: Data on outcome of Stanford type A aortic dissection (TAAD) in Marfan syndrome (MFS) patients are limited. We investigated the full spectrum of reoperation and survival after initial surgery in MFS patients who suffered TAAD.

Methods: Retrospective analysis of 85 consecutive MFS patients in one-single center during the past 15 years.

Results: Overall, 85 MFS patients with TAAD underwent surgical repair [74\% acute dissections; 80\% DeBakey type I; 91\% composite valved graft; 70\% total arch replacement (TAR); 68\% frozen elephant trunk (FET); 7\% in-hospital mortality] at Changhai hospital affiliated to the Second Military Medical University over the past 15 years. Five (20.8\%) patients in non-TAR group need aortic arch reintervention with resternotomy during follow-up, which is significantly higher than that in TAR group $(\mathrm{P}=0.001)$. Freedom from aortic arch reoperation in non-TAR group was all $78.7 \% \pm 8.5 \%$ at 5,10 , and 15 years. No patient required aortic arch reoperation in TAR group $(\mathrm{P}=0.001)$. On the other hand, the FET was inserted into false lumen intentionally at initial surgery in 2 cases of chronic TAAD with narrowed true lumen. Scheduled thoracoabdominal aortic replacement was performed 6 months later. Both 2 patients are with well clinical outcomes. At last, we found that Debakey type and TAR at initial surgery were irrelevant to survival and reoperation for descending aorta.

Conclusions: TAR combined with FET is recommended in MFS patients when the aortic arch is dissected or enlarged. The FET could be inserted into the false lumen intentionally in selective case for scheduled 2-staged descending aortic repair.

Keywords: Marfan syndrome (MFS); type A aortic dissection (TAAD); aortic root surgery; total arch replacement (TAR); frozen elephant trunk (FET)

Submitted Sep 15, 2019. Accepted for publication Nov 19, 2019.

doi: $10.21037 /$ jtd.2019.12.21

View this article at: http://dx.doi.org/10.21037/jtd.2019.12.21

\section{Introduction}

Marfan syndrome (MFS), which is an autosomal-dominant disorder with fibrillin-1 gene mutation, can lead to catastrophic outcome in younger patients (1). Stanford type
A aortic dissection (TAAD) and aneurysm are common pathological manifestations of aorta in these patients. Hence, elective aortic root surgery was used to prevent TAAD and its sequelae. Additionally, prophylactic aortic root procedure made it possible for these patients to achieve 
normal life expectancy.

Limiting surgery to proximal aorta has been proven to be with low mortality in MFS patients with TAAD, while left distal segments untreated $(2,3)$. Consequently, reinterventions for distal aorta, including aortic arch and descending aorta, were necessary in some patients during follow-up. The extent of initial surgery for TAAD in MFS patients was proved to be a determinative factor for reoperation and long-term clinical outcome. Meanwhile, the dissection itself could be a main risk for reintervention $(2,4)$. Hence, some groups advocated that total arch replacement (TAR) combined with frozen elephant trunk (FET) technique was feasible and satisfactory for TAAD in MFS patients, especially in chronic cases (5-8).

This study aimed to present the full spectrum of distal aortic reoperations after initial surgery in MFS patients who suffered TAAD by analyzing a single-center series of 85 patients retrospectively, and to address the controversy that whether distal repair by TAR + FET at initial surgery is feasible and associated with better long-term clinical outcome.

\section{Methods}

\section{Patients and follow-up}

This retrospective analysis was approved by the institutional review board (Project Number: 20180630). The diagnosis of MFS is consistent with the revised Ghent criteria (9). A total of 85 consecutive patients diagnosed with TAAD by contrast-enhanced CT for entire aorta in Changhai hospital between June 2003 and May 2018 were enrolled in this study. The final decision for initial procedure was made by surgeon intraoperatively depending on exploration. Bentall or valve-sparing root replacement (VSRR) technique was alternative for aortic root repair, hemi-arch replacement or TAR was performed for distal repair. FET (MicroPort ${ }^{\circledR}$, Shanghai) was considered when necessary. The indication of TAR + FET for TAAD in MFS patients was DeBakey type I dissection, as well as DeBakey type II dissection with an enlarged arch or proximal descending aorta. The surgical technique of TAR + FET has been depicted in detail previously $(6,10,11)$.

In our center, operative survivors with MFS are routinely followed up at outpatient clinic 3, 6 and 12 months after discharging. After 1 year, the follow-up was performed depending on clinical situation but at least once per year. Transthoracic echocardiography was evaluated annually and contrast-enhanced CT for entire aorta was performed when necessary.

\section{Secondary procedures and definitions}

Late procedures included prosthetic vascular and valvular replacement. In most cases, the indication for prosthetic vascular replacement was residual dissection. Thoracic endovascular aortic repair (TEVAR), open thoracoabdominal aortic replacement, and TAR + FET were performed in subsequent reintervention for diseased aortic segment. The procedure for severe aortic insufficiency was based on recent guidelines and clinical situation.

\section{Statistical analysis}

Continuous variables are presented as mean \pm standard deviation or median (range). Categorical variables are stated as absolute numbers and proportions. Kaplan-Meier analysis was used for evaluation of survival and freedom from reinterventions, and the log-rank test was used to test for differences. The multivariable analysis was performed to determine independent significant prognostic factors. Significant variables associated with reoperation and survival after initial surgery by univariable analysis were included in the multivariable analysis. The statistical analysis was performed by SPSS-V21.0 Software. In all analysis, $\mathrm{P}<0.05$ was considered statistically significant.

\section{Results}

\section{Overall outcomes of initial surgery}

A total of 85 patients were included. The preoperative clinical data at initial surgery are shown in Table 1 . The mean age was 38 years (range, 27-47 years). Fifty-two (61\%) patients were male. Sixty-three $(74 \%)$ cases were acute TAAD and 22 (26\%) cases were chronic one. The preoperative aortic regurgitation grade was severe in 56 $(66 \%)$ cases, moderate in $6(7 \%)$ cases, none and mild in $23(27 \%)$ cases. The entry tear was located in the ascending aorta in $63(74 \%)$ cases, aortic arch in $13(15 \%)$ cases and proximal descending aorta in $9(11 \%)$ cases. It worth mentioning that 8 patients underwent prior Bentall procedure on account of aortic root aneurysm in other hospitals, another 4 cases underwent TEVAR procedure for abdominal aortic aneurysm.

The operative data are shown in Table 2. The times 
Table 1 Preoperative clinical profile

\begin{tabular}{|c|c|}
\hline Variables & $\mathrm{N}=85$ \\
\hline Age, y & $38[27-47]$ \\
\hline Male, $\mathrm{n}[\%]$ & $52[61]$ \\
\hline Years of follow-up, y & $5[3-8]$ \\
\hline Height, cm & $177.3 \pm 9.5$ \\
\hline Weight, kg & $65[59-76]$ \\
\hline \multicolumn{2}{|l|}{ Aortic dissection, $\mathrm{n}[\%]$} \\
\hline Acute & $63[74]$ \\
\hline Chronic & $22[26]$ \\
\hline \multicolumn{2}{|l|}{ Debakey classification, $\mathrm{n}$ [\%] } \\
\hline Type I & $68[80]$ \\
\hline Type II & $17[20]$ \\
\hline $\mathrm{BAV}, \mathrm{n}[\%]$ & $3[4]$ \\
\hline Hypertension, n [\%] & 20 [24] \\
\hline Diabetes mellitus, $\mathrm{n}$ [\%] & $1[1]$ \\
\hline$E F, \%$ & $59 \pm 7$ \\
\hline \multicolumn{2}{|l|}{ Aortic regurgitation, $\mathrm{n}[\%]$} \\
\hline Severe & $56[66]$ \\
\hline Moderate & $6[7]$ \\
\hline None and mild & $23[27]$ \\
\hline Annulus diameter, $\mathrm{cm}$ & $2.5[2.3-2.7]$ \\
\hline Ascending aorta diameter, $\mathrm{cm}$ & $5.2 \pm 1.4$ \\
\hline \multicolumn{2}{|l|}{ Entry tear, n [\%] } \\
\hline Ascending aorta & $63[74]$ \\
\hline Aortic arch & $13[15]$ \\
\hline Proximal descending aorta & $9[11]$ \\
\hline \multicolumn{2}{|c|}{ Coronary artery involvement, n [\%] } \\
\hline Right coronary artery & 10 [12] \\
\hline Left coronary artery & $3[4]$ \\
\hline \multicolumn{2}{|l|}{ History of aortic surgery, $\mathrm{n}$ [\%] } \\
\hline Bentall & 8 [9] \\
\hline TEVAR & $4[5]$ \\
\hline
\end{tabular}

BAV, bicuspid aortic valve; EF, ejection fraction; TEVAR, thoracic endovascular aortic repair.
Table 2 Operative data of initial surgery

\begin{tabular}{|c|c|}
\hline Variables & $\mathrm{N}=85$ \\
\hline \multicolumn{2}{|l|}{ Operative data, min } \\
\hline Cardiopulmonary bypass time & $164.6 \pm 45.5$ \\
\hline Cross-clamp time & $98.9 \pm 28.7$ \\
\hline Cerebral perfusion time & $26.5[22.0-36.5]$ \\
\hline \multicolumn{2}{|l|}{ Proximal repair, n [\%] } \\
\hline Bentall & 77 [91] \\
\hline VSRR & 8 [9] \\
\hline \multicolumn{2}{|l|}{ Distal repair, n [\%] } \\
\hline TAR + FET & $58[68]$ \\
\hline TAR & 2 [2] \\
\hline Non-TAR & $25[30]$ \\
\hline \multicolumn{2}{|l|}{ Concomitant procedures, n [\%] } \\
\hline MVP + TVP & $3[4]$ \\
\hline CABG & $14[16]$ \\
\hline Diameter of FET ,mm & $24.9 \pm 1.5$ \\
\hline \multicolumn{2}{|l|}{ Operative outcomes, n [\%] } \\
\hline Operative mortality & $6[7]$ \\
\hline Spinal cord injury & $0[0]$ \\
\hline Reexploration for bleeding & $7[8]$ \\
\hline Mechanical ventilation time $>72 \mathrm{~h}$ & $12[14]$ \\
\hline Stroke and cerebral hemorrhage & $1[1]$ \\
\hline Renal failure & $5[6]$ \\
\hline
\end{tabular}

V-SARR, valve-sparing aortic root replacement; TAR, total arch replacement; FET, Frozen elephant trunk; MVP, mitral valvuloplasty; TVP, tricuspid valvuloplasty; CABG, coronary artery bypass graft.

of cardiopulmonary bypass, cross-clamp and cerebral perfusion were 164.6 $\pm 45.5,98.9 \pm 28.7,26.5$ (first and third quartiles, 22.0 and 36.5) minutes, respectively. Patients who received Bentall procedure for aortic root repair were in predominant position (77 in 85 patients, $91 \%$ ). TAR + FET was performed in 58 patients (68\%), during which FET was inserted into false lumen intentionally in 2 cases since the true lumen was narrowed (as shown in Figure 1). In 

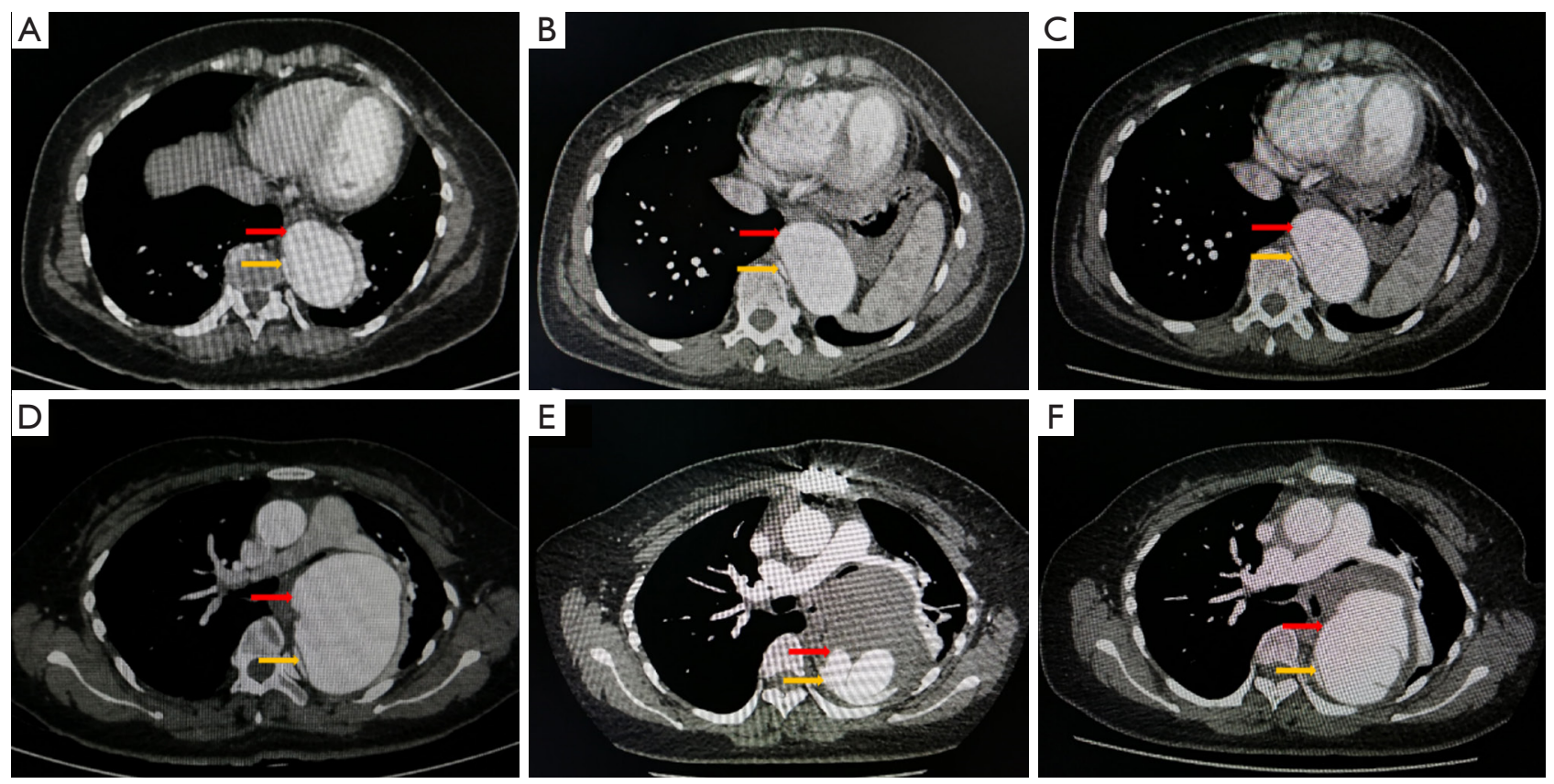

Figure 1 Computed tomography scans of a Marfan syndrome patients with chronic type A aortic dissection before surgery (A,D), 2 weeks after surgery $(\mathrm{B}, \mathrm{E})$, and 6 months $(\mathrm{C}, \mathrm{F})$. The frozen elephant trunk was inserted into false lumen of descending aorta. The upper panel represents the graphs at the diaphragmatic level, the lower panel represents the graphs at the pulmonary artery level. Red arrow represents false lumen; yellow arrow represents narrowed true lumen. The false lumen at the pulmonary artery level narrowed 2 weeks later after surgery (E versus D) and expanded again after 6 months (F versus E).

addition, the aortic diameter at diaphragmatic level in these 2 patients was measured to be $>50 \mathrm{~mm}$, which indicates subsequent procedures were needed during follow-up. Then, scheduled thoracoabdominal aortic replacement was performed 6 months later. Both of these 2 patients are with well clinical outcomes. 2 (2\%) patients in TAR group failed to receive FET implantation because of the distorted morphology of true lumen in descending aorta.

Concomitant procedures included mitral valve surgery in $3(4 \%)$ patients, tricuspid valve surgery in $3(4 \%)$ patients, and coronary artery bypass graft (CABG) in $14(16 \%)$ patients, respectively. Of 14 patients who underwent CABG at initial surgery, 12 patients suffered myocardial ischemia since the coronary artery was involved by TAAD, another 2 patients suffered right ventricular dysfunction when they were off pump. Overall, the operative mortality rate was $7 \%$ (6 of 85 patients). The causes of death were multiorgan failure and sepsis in 4 cases, low cardiac output in 2 cases. Complications included reexploration for bleeding in 7 patients, prolonged ventilation time in 12 patients, renal failure in 5 patients, and cerebral hemorrhage in 1 patient. No spinal cord injury occurred.
Of note, hemodialysis was required in 5 patients who suffered acute renal failure after surgery, of them with poor prognosis. Of 8 patients who had a tracheal intubation time for more than 72 hours, 4 patients died perioperatively. The result of multivariate analysis showed that concomitant $\mathrm{CABG}$ and renal failure were independent risk factors for perioperative death (Table S1).

\section{Reinterventions with secondary sternotomy}

Six patients underwent 6 re-sternotomies for prosthetic vascular or valvular replacement. Five patients underwent secondary TAR + FET after proximal repair at initial surgery. One patient died perioperatively on account of multiorgan failure, and another 1 died of distal aortic rupture before discharging. The in-hospital mortality rate for secondary TAR was 40\% ( 2 of 5 patients). Additionally, 1 patient underwent re-sternotomy for mitral valve replacement 14 years after initial surgery. No aortic valve reintervention procedure was needed in both VSRR and Bentall group.

Secondary TAR + FET became necessary in 5 of 


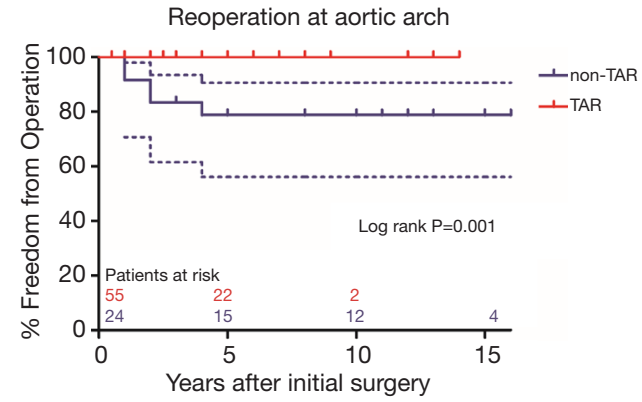

Figure 2 Freedom from reintervention in aortic arch in Marfan syndrome patients with TAAD who underwent TAR versus nonTAR at initial surgery. TAAD, type A aortic dissection; TAR, total arch replacement.

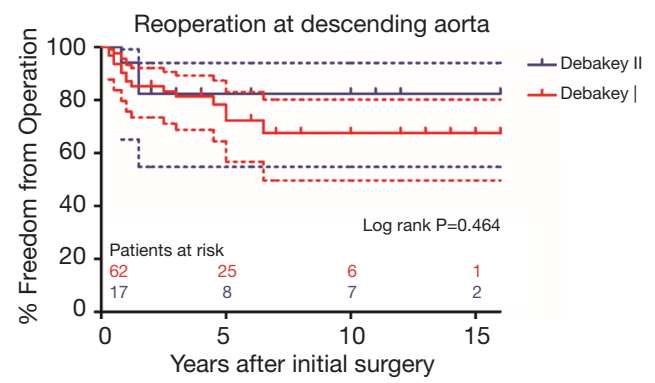

Figure 4 Freedom from reintervention in descending aorta in Marfan syndrome patients with TAAD stratified by Debakey classification. TAAD, type A aortic dissection.

24 patients $(20.8 \%)$ in non-TAR group, while no patient required secondary distal repair in TAR group $(\mathrm{P}=0.001)$. Freedom from aortic arch reoperation in non-TAR group were all $78.7 \% \pm 8.5 \%$ at 5,10 , and 15 years (Figure 2). Of note, 10 cases classified into Debakey I group failed to undergo TAR procedure at initial surgery due to their poor general condition, while 2 cases classified into Debakey II group underwent TAR + FET for the enlarged aortic arch. Similarly, no patient required secondary aortic arch surgery in Debakey I group, and secondary TAR + FET was necessary in 5 of 17 patients (29.4\%) in Debakey II group. As shown in Figure 3, freedom from aortic arch reoperation in Debakey II group were all $68.8 \% \pm 11.8 \%$ at 5,10 , and 15 years $(\mathrm{P}<0.001)$.

\section{Reinterventions for descending aorta}

Eighteen patients underwent 24 procedures on downstream aortic segments during follow-up, including 21 cases

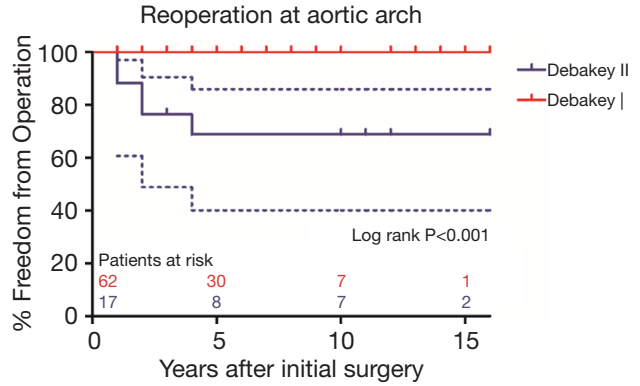

Figure 3 Freedom from reintervention in aortic arch stratified by Debakey classification.

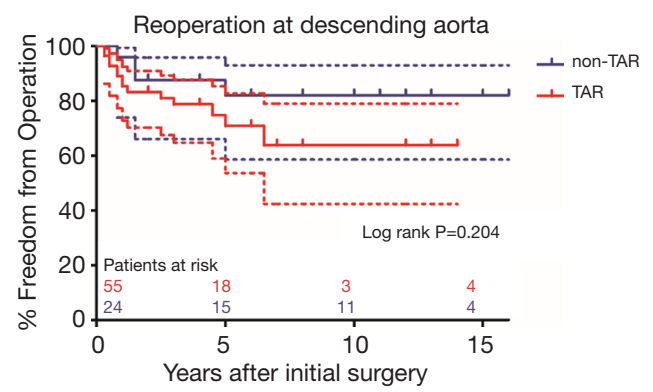

Figure 5 Freedom from reintervention in descending aorta in Marfan syndrome patients with TAAD who underwent TAR versus non-TAR at initial surgery. TAR, total arch replacement; TAAD, type A aortic dissection.

of TEVAR and 3 cases of thoracoabdominal aortic replacement. Of these 18 patients, 4 patients underwent more than 1 operation. The most common indication for reintervention was residual dissection. Aortic aneurysm was another indication in 5 patients. No patient died perioperatively in secondary operation for descending aorta. One patient underwent TEVAR died of massive hemorrhage for aortic esophageal fistula 6 years later, and a second patient received thoracoabdominal aortic replacement died of stent infection 19 months after discharging.

As shown in Figure 4, freedom from descending aortic reoperation in patients with DeBakey type I dissection were $78.3 \% \pm 5.7 \%, 67.4 \% \pm 7.8 \%$, and $67.4 \% \pm 7.8 \%$ and in patients with DeBakey type II dissection were all $82.4 \% \pm 9.2 \%$ at 5,10 , and 15 years, respectively $(\mathrm{P}=0.464)$. Freedom from descending aortic reoperation in non-TAR group were all $82.0 \pm 8.3 \%$ and in TAR group were $70.9 \% \pm 7.4 \%, 63.8 \% \pm 9.5 \%, 63.8 \% \pm 9.5 \%$ 


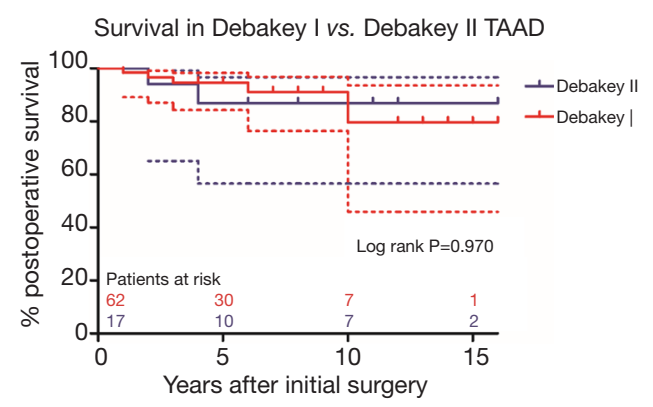

Figure 6 Survival of Marfan syndrome patients with Debakey I and Debakey II TAAD. TAAD, type A aortic dissection.

at 5,10 , and 15 years, respectively as shown in Figure 5 $(\mathrm{P}=0.204)$.

\section{Survival}

During follow-up, cerebral hemorrhage with poor outcome occurred in 1 patient half year after discharging. 1 patient died of respiratory failure 13 years later. Two patients died perioperatively at secondary aortic arch operation as mentioned before. Two patients died after reintervention for descending aorta. Another 3 patients died for unknown reason.

Survival in patients with DeBakey type I dissection were $94.7 \% \pm 3.0 \%, 79.6 \% \pm 11.4 \%$, and in patients with DeBakey type II dissection were $86.9 \% \pm 8.7 \%, 86.9 \% \pm 8.7 \%$ at 5, 10 years, respectively (Figure 6, $\mathrm{P}=0.970$ ). Survival in non-TAR group were $91.3 \% \pm 5.9 \%, 84.2 \% \pm 8.7 \%$, and in TAR group were $93.9 \% \pm 3.4 \%, 89.2 \% \pm 5.6 \%$ at 5,10 years, respectively (Figure $7, \mathrm{P}=0.980$ ).

\section{Discussion}

The indications for subsequent procedure in MFS patients after initial surgery are mainly the pathological changes in remaining native aorta, followed by the changes in aortic and mitral valve (12). Hence, the extent of initial surgery, which keep controversy, may determine the longterm outcome of MFS patients presenting with TAAD. Some groups have reported that a more aggressive surgical strategy seems to be superior to proximal repair at initial surgery for TAAD in MFS patients $(4,6,7)$, so as to reduce the risk of subsequent reintervetion. TAR combined with FET technique may facilitate stabilizing and remodelling distal aorta, then was verified to be satisfactory in MFS patients with TAAD $(6,7)$. In this paper, we report the

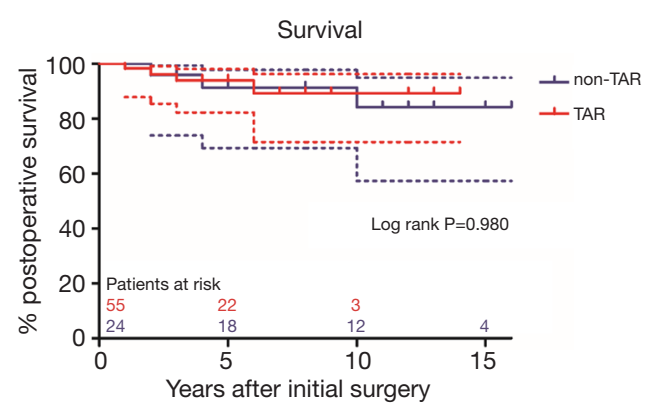

Figure 7 Survival of Marfan syndrome patients with TAR and non-TAR at initial surgery. TAR, total arch replacement.

results of our series of MFS patients presenting with TAAD.

As for aortic root repair, patients who received Bentall procedure were in predominant position in our study. None of these patients needs reintervention for aortic root during follow-up. Meanwhile, thromboembolism and endocarditis events related to mechanical valve were absent, except for 1 patient suffered from cerebral bleeding. Bentall procedure has been verified to be a reliable and durable solution for MFS patients presenting with TAAD. Nicolo et al. suggested that Bentall procedure would continue to be a standard treatment for elective aortic root replacement in MFS patients (13). In contrast, other groups advocated the notion that VSRR is a feasible alternative for Bentall by balancing the risk of root reoperation and the benefit of exemption for complications related to mechanical valves, and comparing the late survival (14-19). VSRR has certainly become the first choice for MFS patients presenting with aneurysm in our center over the past decade. Meanwhile, VSRR was also used for aortic root repair in MFS patients with TAAD recently. However, we still keep a conservative attitude toward VSRR when referred to the MFS patients with TAAD for our limited experience.

Whether TAR should be performed at initial surgery in MFS patients is controversial. Some groups advocated the notion that the extent of surgery for TAAD should be limited into ascending aortic replacement or proximal aortic arch for its low perioperative mortality, the aortic arch repair can be performed electively afterwards with low mortality (20). On the contrary, Bachet et al. reported that secondary TAR was required in $73 \%$ MFS patients with TAAD after Bentall procedure, then proposed a more aggressive approach toward the aortic arch at initial surgery (21). Sun et al. reported that TAR + FET was in superior position for TAAD $(6,10)$. Subsequently, such technique was confirmed to be suitable for patients with 
MFS with chronic aortic dissection (5).

In our series, distal repair by TAR + FET is utilized for TAAD in MFS patients whose aortic arch is dissected or enlarged, especially in patients with Debakey type I TAAD. Low perioperative mortality, satisfactory long-term survival, and free from reoperation in aortic arch were achieved in TAR group, which is consist with the result in Ma's work (6). In addition, the physical condition of MFS patients allows for a longer operative time since aortic dissection occurs on average 20 years earlier in these patients compared with peers without MFS. Most importantly, secondary TAR + FET may with a higher in-hospital mortality rate, which is similar to the previous work (4). Therefore, we believe that a more radical approach may be justified in MFS patients presenting with TAAD when the aortic arch is dissected or enlarged. Proximal repair could be reasonable for MFS patients with Debakey type II TAAD, while regular imaging surveillance should be indispensable during follow-up.

Aortic dissection extended beyond the aortic arch at the time of initial surgery was associated with higher reoperation rates on descending aorta (4). Sun et al. observed that FET + TAR group required less reintervention and had a higher rate of false lumen thrombosis (10). Hence, FET + TAR may provide an alternative way for DeBakey type I dissection, and reduce the risk of reoperation for descending aorta. Of note, FET can reduce the complexity in late descending aortic operation by clamping of the elephant trunk without deep hypothermia (22). In our series, 2 cases of chronic TAAD have developed thoracoabdominal aneurysm, both of them with narrowed true lumen. The FET was inserted into false lumen intentionally at initial surgery, then scheduled thoracoabdominal aortic replacement was performed 6 months later. Both of these 2 patients are with well clinical outcomes.

Previous studies have reported excellent short-term and long-term effects of 2-staged thoracoabdominal aortic replacement in patients with or without MFS $(23,24)$. Roselli et al. found that staged repair strategies, including open thoracoabdominal aortic replacement and TEVAR, are feasible and benefit outweigh risks (25). Marcheix observed that TEVAR is feasible in selected MFS patients with low mortality and morbidity rates. However, lifelong imaging surveillance is crucial since the rate of endoleak is high (26). Based on our experience and that of others, we believe distal repair at initial surgery and selective 2-staged descending aortic repair may feasible for MFS patients with TAAD.

\section{Limitation}

This was a retrospective study conducted in a small number of patients, and all operations were performed in a single institute. Patients selected for analysis were operated on within the past 15 years. During that time period, therapeutic regimens were likely to have changed, potentially influencing the results.

\section{Conclusions}

Bentall procedure was feasible for its low rate of valve prosthesis-related complications and satisfactory longterm results in MFS with TAAD. TAR combined with FET is recommended in MFS patients when the aortic arch is dissected or enlarged since the risk of secondary TAR can be avoided. The FET could be inserted into the false lumen intentionally in selective patients for facilitating scheduled 2-stage thoracoabdominal aortic replacement. The TEVAR and thoracoabdominal aortic replacement could be recommended for descending aortic repair in selected MFS patients.

\section{Acknowledgments}

Funding: This study was funded by Shanghai Committee of Science and Technology, China (Grant No. 2014ZYJB0401).

\section{Footnote}

Conflicts of Interest: The authors have no conflicts of interest to declare.

Ethical Statement: The authors are accountable for all aspects of the work in ensuring that questions related to the accuracy or integrity of any part of the work are appropriately investigated and resolved. This retrospective analysis was approved by the institutional review board (Project Number: 20180630).

\section{References}

1. Rylski B, Hoffmann I, Beyersdorf F, et al. Acute aortic dissection type A: age-related management and outcomes reported in the German Registry for Acute Aortic Dissection Type A (GERAADA) of over 2000 patients. 
Ann Surg 2014;259:598-604.

2. Schoenhoff FS, Kadner A, Czerny M, et al. Should aortic arch replacement be performed during initial surgery for aortic root aneurysm in patients with Marfan syndrome? Eur J Cardiothorac Surg 2013;44:346-51; discussion 351 .

3. Girdauskas E, Kuntze T, Borger MA, et al. Distal aortic reinterventions after root surgery in Marfan patients. Ann Thorac Surg 2008;86:1815-9.

4. Rylski B, Bavaria JE, Beyersdorf F, et al. Type A aortic dissection in Marfan syndrome: extent of initial surgery determines long-term outcome. Circulation 2014;129:1381-6.

5. Sun L, Li M, Zhu J, et al. Surgery for patients with Marfan syndrome with type A dissection involving the aortic arch using total arch replacement combined with stented elephant trunk implantation: the acute versus the chronic. J Thorac Cardiovasc Surg 2011;142:e85-91.

6. Ma WG, Zhang W, Zhu JM, et al. Long-term outcomes of frozen elephant trunk for type A aortic dissection in patients with Marfan syndrome. J Thorac Cardiovasc Surg 2017;154:1175-1189.e2.

7. Chen Y, Ma WG, Zhi AH, et al. Fate of distal aorta after frozen elephant trunk and total arch replacement for type A aortic dissection in Marfan syndrome. J Thorac Cardiovasc Surg 2018. [Epub ahead of print].

8. Sun L, Qi R, Chang Q, et al. Surgery for marfan patients with acute type a dissection using a stented elephant trunk procedure. Ann Thorac Surg 2008;86:1821-5.

9. Loeys BL, Dietz HC, Braverman AC, et al. The revised Ghent nosology for the Marfan syndrome. J Med Genet 2010;47:476-85.

10. Sun L, Qi R, Zhu J, et al. Total arch replacement combined with stented elephant trunk implantation: a new "standard" therapy for type a dissection involving repair of the aortic arch? Circulation 2011;123:971-8.

11. Chen Y, Ma WG, Zheng J, et al. Total arch replacement and frozen elephant trunk for type A aortic dissection after Bentall procedure in Marfan syndrome. J Thorac Dis 2018;10:2377-87.

12. Puluca N, Burri M, Cleuziou J, et al. Consecutive operative procedures in patients with Marfan syndrome up to 28 years after initial aortic root surgery. Eur J Cardiothorac Surg 2018;54:504-9.

13. Nicolo F, Romeo F, Lio A, et al. Long-Term Results of Aortic Root Surgery in Marfan Syndrome Patients: A
Single-Center Experience. J Heart Valve Dis 2017;26:397-404.

14. Schoenhoff FS, Langhammer B, Wustmann K, et al. Decision-making in aortic root surgery in Marfan syndrome: bleeding, thromboembolism and risk of reintervention after valve-sparing or mechanical aortic root replacement. Eur J Cardiothorac Surg 2015;48:931-5; discussion 935-6.

15. Mosbahi S, Stak D, Gravestock I, et al. A systemic review and meta-analysis: Bentall versus David procedure in acute type A aortic dissection. Eur J Cardiothorac Surg 2019;55:201-9.

16. Shrestha ML, Beckmann E, Abd Alhadi F, et al. Elective David I Procedure Has Excellent Long-Term Results: 20-Year Single-Center Experience. Ann Thorac Surg 2018;105:731-8.

17. Tanaka H, Ogino H, Matsuda H, et al. Midterm outcome of valve-sparing aortic root replacement in inherited connective tissue disorders. Ann Thorac Surg 2011;92:1646-9; discussion 1649-50.

18. David TE, David CM, Feindel CM, et al. Reimplantation of the aortic valve at 20 years. J Thorac Cardiovasc Surg 2017;153:232-8.

19. Coselli JS, Volguina IV, LeMaire SA, et al. Early and 1 -year outcomes of aortic root surgery in patients with Marfan syndrome: a prospective, multicenter, comparative study. J Thorac Cardiovasc Surg 2014;147:1758-66, 1767.e1751-4.

20. Rylski B, Beyersdorf F, Kari FA, et al. Acute type A aortic dissection extending beyond ascending aorta: Limited or extensive distal repair. J Thorac Cardiovasc Surg 2014;148:949-54; discussion 954.

21. Bachet J, Larrazet F, Goudot B, et al. When should the aortic arch be replaced in Marfan patients? Ann Thorac Surg 2007;83:S774-9; discussion S785-90.

22. Etz CD, Plestis KA, Kari FA, et al. Staged repair of thoracic and thoracoabdominal aortic aneurysms using the elephant trunk technique: a consecutive series of 215 first stage and 120 complete repairs. Eur J Cardiothorac Surg 2008;34:605-14; discussion 614-5.

23. Castrovinci S, Murana G, de Maat GE, et al. The classic elephant trunk technique for staged thoracic and thoracoabdominal aortic repair: long-term results. J Thorac Cardiovasc Surg 2015;149:416-22.

24. Ikeno Y, Yokawa K, Nakai H, et al. Results of staged repair of aortic disease in patients with Marfan syndrome. J Thorac Cardiovasc Surg 2019;157:2138-2147.e2. 
25. Roselli EE, Idrees JJ, Lowry AM, et al. Beyond the Aortic Root: Staged Open and Endovascular Repair of Arch and Descending Aorta in Patients With Connective Tissue Disorders. Ann Thorac Surg 2016;101:906-12.

Cite this article as: Li N, Zhang Y, Gao Y, Bai Y, An Z, Zhang G, Han Q, Lu F, Li B, Han L, Xu Z. Decision-making at initial surgery for type A aortic dissection in patients with Marfan syndrome: proximal or extensive repair. J Thorac Dis 2019;11(12):4951-4959. doi: 10.21037/jtd.2019.12.21
26. Marcheix B, Rousseau H, Bongard V, et al. Stent grafting of dissected descending aorta in patients with Marfan's syndrome: mid-term results. JACC Cardiovasc Interv 2008;1:673-80. 
Supplementary

Table S1 Multivariate analysis for evaluating predictors of survival

\begin{tabular}{lcc}
\hline Variable & OR & P value \\
\hline Acute TAAD & 0.941 & 0.166 \\
Debakey type II & 0.969 & 0.242 \\
Concomitant CABG & 0.054 & 0.017 \\
Cross-clamp time & 4.564 & 0.179 \\
Mechanical ventilation time $>72 \mathrm{~h}$ & 0.958 & 0.188 \\
Renal failure & 0.009 & $<0.001$ \\
\hline
\end{tabular}

TAAD, type A aortic dissection; CABG, coronary artery bypass graft. 